\title{
Normal pressure hydrocephalus co-existing with a second dementia disorder
}

\author{
Joseph Alisky ${ }^{1,2}$ \\ 'Marshfield Clinic Research \\ Foundation, 1000 Oak Avenue, \\ Marshfield,Wisconsin, USA; \\ 2Marshfield Clinic Thorp Center, 704 \\ South Clark, Thorp, Wisconsin, USA
}

\begin{abstract}
An 87-year-old woman had both Lewy body dementia and normal pressure hydrocephalus simultaneously. One should not automatically exclude the possibility of normal pressure hydrocephalus simply because another neurodegenerative disorder is present, especially if the second disorder masks typical symptoms of normal pressure hydrocephalus. Better identification of dual diagnosis patients could lead to improvements in gait and incontinence following ventriculoperitoneal or lumboperitoneal shunting, even if the natural history of the other neurodegenerative disease remains unchanged.
\end{abstract}

Keywords: dementia, gait disorder, normal pressure hydrocephalus, Lewy body dementia, ventriculoperitoneal shunt

\section{Case report}

An 85-year-old woman manifested a gait disorder, urinary incontinence and mild cognitive impairment. A magnetic resonance image (MRI) of the brain showed ventriculomegaly consistent with normal pressure hydrocephalus, and her gait improved after a large volume lumbar puncture (removal of $50 \mathrm{ml}$ of cerebrospinal fluid). She was recommended to have a ventriculoperitoneal shunt placed, but she declined the procedure and was lost to follow-up. Two years later, at age 87 , she was hospitalized because of constant falling episodes. She was unable to stand independently and had much worse problems with memory and recall, accompanied by waxing and waning mental status, mild rigidity and occasional visual hallucinations. Based on these new symptoms, a diagnosis of Lewy body dementia was made, and she was placed in a nursing home, at which point I first assumed her care. I had the patient undergo another large volume lumbar puncture and she again markedly improved her gait. Falling episodes ceased and she was able to walk on her own. She was started on the cholinesterase inhibitor donepezil $5 \mathrm{mg}$ daily, which was increased to $10 \mathrm{mg}$ after a month for treatment of the Lewy body dementia. Donepezil eliminated almost all confusion and hallucinations. She then agreed to have a ventriculoperitoneal shunt placed. Postoperatively, she suffered a deep venous thrombosis but subsequently has made a good recovery. At the time of this writing, post-ventriculoperitoneal shunting and continuing on donepezil, she is ambulating on her own with only minimal need for assistance and has left the nursing home for an assisted-living facility where she has her own apartment, but the facility does the housework, provides meals, and sets out her medications. Her mental status is now excellent. Her score on the Folstein mini-mental status exam is now 29/30 compared with 15/30 before the cholinesterase inhibitor and ventriculoperitoneal shunt.

\section{Overview of normal pressure hydrocephalus}

Normal pressure hydrocephalus is characterized by hydrocephalus with normal intracranial pressure, gait disorder, incontinence, and cognitive impairment. 
The pathophysiology of normal pressure hydrocephalus is poorly understood and is believed to have something to do with decreased absorption of cerebrospinal fluid rather than over-production. If there is obstruction of cerebrospinal fluid, by definition normal pressure hydrocephalus is not present. Definitive treatment is placement of a ventriculoperitoneal shunt to drain excess cerebrospinal fluid out of the central nervous system (Verrees and Selman 2004). As reviewed in detail by Relkin and colleagues (2005), the likelihood of normal pressure hydrocephalus being present can be stratified as probable, possible, and unlikely, depending on history, physical findings, and supporting studies (Relkin et al 2005).

Probable normal pressure hydrocephalus requires insidious slow onset in someone over 40 years old, with symptoms present for 3-6 months, worsening over time, and with no other neurological or other condition that can account for the presentation and no known head trauma, cerebrovascular accident or other brain injury. Brain imaging, either computer axial tomography (CAT) or MRI, should show ventricular atrophy not caused by cerebral atrophy or congential conditions. There should be no observable obstruction to normal flow of cerebrospinal fluid and at least 1 of 4 features should be seen in the brain images: enlargement of temporal horns of the lateral ventricles that cannot be explained by hippocampal atrophy, a callosal angle over 40 degrees, evidence of altered brain parenchyma such as periventricular signal changes that cannot be explained by microvascular disease or demyelination, and a flow void in the cerebral aqueducts or 4 th ventricle seen on MRI. A patient must manifest a gait disturbance, with at least 2 of 9 features present: decreased step height, diminished step length, slowed walking, truncal swaying during walking, a widened gait, toes deviating outward, spontaneous retropulsion, difficulty turning (patients need three or more steps to turn a half-circle), and difficulty keeping balance while walking, as manifested by two or more corrections during eight steps of tandem walking. In addition to these brain imaging findings and the gait disturbance, to make a diagnosis of probable normal pressure hydrocephalus, a patient must show cognitive impairment and urinary or fecal incontinence. Cognitive impairment can be documented by either neuropsychological testing or alternatively by the presence of at least 2 out of 7 cardinal symptoms of cognitive impairment: psychomotor slowing, bradykinesia, poor coordination, difficulty with attention, impaired recall, loss of executive functioning, and significant alterations in behavior and personality. Finally there should be a normal cerebrospinal fluid opening pressure on lumbar puncture in the range 5-18 mm of mercury (Relkin et al 2005).
To diagnose possible normal pressure hydrocephalus, the criteria are simpler and less stringent. There should be a gradual onset of symptoms at any age after childhood. Symptoms do not need to be present for 6 months; 3 months or less or unknown duration still meets the requirement. Possible normal pressure hydrocephalus can be subsequent to mild head trauma, past intracranial bleeding, meningitis or other brain disease not thought to be responsible for present symptoms, and possible normal pressure hydrocephalus can co-exist with other brain disorders if the examining clinician feels that these other disorders are not the proximate cause of symptoms. Brain imaging should show ventriculomegaly, but there can also be evidence of cerebral atrophy and structural lesions present. Patients should manifest either incontinence and cognitive impairment or gait disturbance or dementia alone, without incontinence (Relkin et al 2005).

Normal pressure hydrocephalus is felt to be unlikely if ventriculomegaly is not present, if there is an absence of any component of the normal pressure hydrocephalus triad (dementia, incontinence, and gait disorder), if there is elevated intracranial pressure (as manifested by papilledema or high opening pressure) and finally if symptoms can be accounted for by another neurological disorder (Relkin et al 2005).

As an alternative to the above criteria, some studies have shown that improvement in gait, cognition, and continence following a large volume lumbar puncture (taking off $50 \mathrm{ml}$ of cerebrospinal fluid) can identify patients who will have a good response to a permanent ventriculoperitoneal shunt (Wikkelso et al 1986; Walchenbach et al 2002; Verrees and Selman 2004). This was the approach followed for my patient. Correlation between improvement after lumbar puncture and ventriculoperitoneal shunting is good but not perfect. There still are patients who would not improve after the spinal tap but still would improve after implantation of a shunt (Walchenbach et al 2002).

If ventriculoperitoneal shunting presents too high an operative risk in frail elderly persons, percutaneous lumboperitoneal shunting done under local anesthesia would provide another therapeutic option. There are studies showing that lumboperitoneal shunts can be effective for normal pressure hydrocephalus (Spetzler et al 1977; Selman et al 1980; Yadav et al 2004) but this is not a commonly used approach. A neurosurgeon I spoke with said that he thought lumboperitoneal shunts would be prone to failure, and in the United States, one would have to make a special search to find surgeons or interventional radiologists who implant lumboperitoneal shunts for normal pressure hydrocephalus. Still, the lumboperitoneal shunt should remain on the table as 
at least an option when medical comorbidities make operative placement of a ventriculoperitoneal shunt unacceptable.

\section{Lewy body dementia}

The second disorder of this report, Lewy body dementia, is very common among the elderly, probably only behind Alzheimer's disease in frequency. Diagnosis of probable Lewy body dementia can be made if patients have fluctuating mental status, visual hallucinations, and mild parkinsonism, although unequivocal identification would require finding characteristic Lewy body inclusions within neurons via either brain biopsy or postmortem examination (McKeith 2002; McKeith et al 2005). The visual hallucinations and waxing mental status often improve with the addition of a cholinesterase inhibitor, while parkinsonism of Lewy body dementia sometimes responds to dopaminergic agents (McKeith et al 2005; Molloy et al 2005).

\section{Implications for practice and research}

The patient described here first presented with normal pressure hydrocephalus and later with Lewy body dementia. When she had only normal pressure hydrocephalus, her mental status was almost normal, but later on more serious cognitive dementia and fluctuating mental status from Lewy body dementia superseded any cognitive impairment originating from the original normal pressure hydrocephalus. Similarly, the original shuffling gait from normal pressure hydrocephalus was subsequently swamped out by frequent falling episodes secondary to parkinsonism of Lewy body dementia. How common is normal pressure hydrocephalus superimposed with a second disorder? At this point, available data is only anecdotal, consisting of previously published histories of normal pressure hydrocephalus co-existing with Parkinson's disease, vascular dementia, and Alzheimer's disease (Kruger 1982; Lobo Antunes et al 1983; Muramatsu et al 2000; Nakabayashi et al 1984; Golomb et al 2000; Silverberg et al 2006). In all of the earlier reports, gait could improve after shunting but the natural history of the other disorder was unchanged. For example, the patients with Parkinson's disease had amelioration of normal pressure hydrocephalus-related gait problems following shunting, but subsequently a typical parkinsonian presentation dominated the picture (Kruger 1982; Lobo Antunes et al 1983).

Better knowledge of how often normal pressure hydroephalus co-occurs with other dementia disorders could have important clinical significance. Given that gait disorders are very common in the later stages of virtually all types of dementia, I propose the hypothesis that other dementia syndromes may often obscure the diagnosis of normal hydrocephalus. Furthermore, neurodegenerative diseases may also actually cause normal pressure hydrocephalus later in their courses from damage to parenchymal brain tissue. This is certainly known to be the case for patients who have suffered head injuries and cerebrovascular accidents (Beyerl and Black 1984; Nakayabashi et al 1984), so it would not be unreasonable to posit a similar phenomenon in the setting of chronic progressive neurodegenerative diseases. As with stroke and head injury, there is physical damage to brain tissue (consider the profound cortical atrophy in Alzheimer's disease), and this physical damage may alter cerebrospinal fluid mechanics. If so, one might not see a typical normal pressure hydrocephalus picture, but patients might still improve after shunting with corresponding better quality of life and perhaps less expensive care. Furthermore, because of the second disorder these cases would be classified as possible rather than probable normal pressure hydrocephalus by Relkin's criteria (Relkin et al 2005), meaning one would have to have a higher index of suspicion to proceed with ventriculoperitoneal shunting. Practicing clinicians should be liberal about ordering diagnostic large volume lumbar punctures or imaging studies for patients with dementia, incontinence, and gait disorders, even when a likely normal pressure hydrocephalus picture is not present. Along the same lines, researchers should conduct open clinical trials, doing lumbar punctures or imaging studies on dementia patients with gait disorders, with as many as possible undergoing ventriculoperitoneal or lumboperitoneal shunting. This idea is thus put forward in Neuropsychiatric Disease and Treatment in the hopes it can go forward.

\section{References}

Beyerl B, Black PM. 1984. Posttraumatic hydrocephalus. Neurosurgery, 15:257-61.

Golomb J, Wisoff J, Miller DC, et al. 2000. Alzheimer's disease comorbidity in normal pressure hydrocephalus: prevalence and shunt response. J Neurol Neurosurg Psychiatry, 68:778-81.

Kruger LB. 1982. Normal pressure hydrocephalus in the parkinsonian patient. J Neurosurg Nurs, 14:299-302.

Lobo Antunes J, Fahn S, Cote L. 1983. Normal pressure hydrocephalus and Parkinson's disease. J Neural Transm Suppl, 19:225-31.

McKeith IG. 2002. Dementia with Lewy bodies. Br J Psychiatry, 180:144-7.

McKeith IG, Dickson DW, Lowe J, et al. 2005. Diagnosis and management of dementia with Lewy bodies: third report of the DLB Consortium. Neurology, 65:1863-72.

Molloy S, McKeith IG, O'Brien JT, et al. 2005. The role of levodopa in the management of dementia with Lewy bodies. J Neurol Neurosurg Psychiatry, 76:1200-3. 
Muramatsu H, Nathan RD, Shimura T, et al. 2000. Recovery of stroke hemiplegia through neurosurgical intervention in the chronic stage. NeuroRehabilitation, 15:157-66.

Nakabayashi S, Uyama O, Yoneda S, et al. 1984. [A case of normal pressure hydrocephalus associated with cerebrovascular disorders] [Article in Japanese]. No To Shinkei, 36:1077-82.

Relkin N, Marmarou A, Klinge P, et al. 2005. Diagnosing idiopathic normalpressure hydrocephalus. Neurosurgery, 57(3 Suppl):S4-16.

Selman WR, Spetzler RF, Wilson CB, et al. 1980. Percutaneous lumboperitoneal shunt: review of 130 cases. Neurosurgery, 6:255-7.

Silverberg G, Mayo M, Saul T, et al. 2006. Elevated cerebrospinal fluid pressure in patients with Alzheimer's disease. Cerebrospinal Fluid Res, 3:7.

Spetzler R, Wilson CB, Schulte R. 1977. Simplified percutaneous lumboperitoneal shunting. Surg Neurol, 7:25-9.
Verrees M, Selman WR. 2004. Management of normal pressure hydrocephalus. Am Fam Physician, 70:1071-8.

Walchenbach R, Geiger E, Thomeer RT, et al. 2002. The value of temporary external lumbar CSF drainage in predicting the outcome of shunting on normal pressure hydrocephalus. J Neurol Neurosurg Psychiatry, 72:503-6.

Wikkelso C, Andersson H, Blomstrand C, et al. 1986. Normal pressure hydrocephalus. Predictive value of the cerebrospinal fluid tap-test. Acta Neurol Scand, 73:566-73.

Yadav YR, Pande S, Raina VK, Singh M. 2004. Lumboperitoneal shunts: review of 409 cases. Neurol India, 52:188-90. 